01

\title{
Расчет релятивистских штарковских энергий и ширин водородоподобных ионов методом комплексного вращения
}

\author{
(C) И.А. Мальцев ${ }^{1}$, Д.А. Тумаков ${ }^{1,2}$, Р.В. Попов ${ }^{1}$, В.М. Шабаев ${ }^{1}$ \\ ${ }^{1}$ Санкт-Петербургский государственный университет, \\ 199034 Санкт-Петербург, Россия \\ ${ }^{2}$ ФТИ им. А.Ф. Иоффре РАН, \\ 194021 Санкт-Петербург, Россия \\ e-mail: i.maltsev@spbu.ru, d.tumakov@spbu.ru
}

Поступила в редакцию 30.12.2021 г.

В окончательной редакции 30.01.2021 г.

Принята к публикации 10.01.2021 г.

Выполнены релятивистские расчеты энергий и ширин нижних состояний атома водорода и водородоподобного иона неона. Расчеты проведены с помощью техники конечного базисного набора в комбинации с методом комплексного вращения. Полученные результаты сравниваются с доступными в литературе нерелятивистскими и релятивистскими значениями.

Ключевые слова: эффект Штарка, комплексное вращение.

DOI: $10.21883 /$ OS.2022.04.52278.3113-21

\section{Введение}

Связанные состояния атома или иона, помещенного в однородное электрическое поле, сдвигаются и превращаются в резонансы, имеющие ненулевую ширину и, как следствие, конечное время жизни. Данное явление называется эффектом Штарка и изучалось долгое время как экспериментально [1-6], так и теоретически [7-28]. Однако большая часть теоретических работ посвящена нерелятивистским расчетам. Необходимо отметить, что релятивистские эффекты могут иметь значение уже в случае атома водорода [23], а для тяжелых ионов только релятивистские расчеты могут дать точные результаты. При этом прецизионные эксперименты с тяжелыми многозарядными ионами в очень сильных электрических полях станут возможными в ближайшем будущем. Например, такие экспериментальные исследования будут осуществимы в рамках проекта Gamma Factory [29]. Ожидается, что посредством эффекта Штарка можно будет манипулировать уровнями тяжелых ионов заданным образом. Точные теоретические значения положений этих уровней представляются крайне важными для таких исследований. Кроме того, значения ширин уровней необходимы для оценки стабильности пучков ионов в сильных магнитных полях.

Релятивистские расчеты положений штарковских резонансов с помощью теории возмущений были выполнены в работе [8]. В статье [28] использовался конечный базисный набор для расчета релятивистского штарковского сдвига в атоме водорода и водородоподобном аргоне. Стоит отметить, что точность методов, основанных на представлении эрмитова гамильтониана в конечном базисе, ограничена, так как волновые функции резонансов не являются квадратично интегрируемыми в отличие от базисных функций. Кроме того, такие методы не позволяют получить ширину резонанса (вероятность ионизации в единицу времени). Релятивистская ширина штарковских резонансов для водородоподобных ионов была получена в работах $[21,22]$ с помощью квазиклассического приближения. Точность такого приближения, однако, также ограничена.

Высокоточные значения как положения резонанса, так и его ширины могут быть получены с помощью метода комплексного вращения. Данный метод основан на повороте радиальной координаты в комплексную плоскость. После поворота резонансные волновые функции становятся квадратично интегрируемыми, что позволяет использовать стандартные методы, основанные на использовании конечного базисного набора. При этом гамильтониан теряет эрмитовость, и его собственные энергии, соответствующие резонансам, принимают комплексные значения. Действительная часть такой комплексной энергии соответствует положению резонанса, а мнимая часть определяет его ширину. Релятивистский метод комплексного вращения ранее успешно использовался для расчета автоионизационных состояний многоэлектронных ионов [30-33] и параметров сверхкритического резонанса в тяжелых квазимолекулах [34-37]. Описание метода комплексного вращения, его различных вариаций и приложений можно найти в обзорах $[38,39]$.

Для расчета штарковских энергий и ширин релятивистский метод комплексного вращения применялся в работе [23]. Расчеты были выполнены для нижних состояний атома водорода и водородоподобного иона неона. На основе полученных результатов был сделан вывод о значимости релятивистских эффектов даже для водорода, особенно при относительно малых значениях напряженности электрического поля. При этом было 
выявлено, что с ослаблением электрического поля релятивистские значения для ширины начинают превосходить нерелятивистские. Этот результат качественно отличается от вывода работ [21,22], основанного на квазиклассических расчетах, согласно которому при малых значениях напряженности электрического поля релятивистские значения ширины меньше нерелятивистских.

Цель представленной работы - разрешить имеющееся противоречие в теоретических результатах и исследовать влияние релятивистских эффектов на положения и ширины штарковских резонансов. С этой целью были проведены релятивистские расчеты для атома водорода и водородоподобного иона неона. Расчеты были выполнены в конечном базисном наборе с использованием метода комплексного вращения. Полученные результаты были сопоставлены с соответствующими значениями из работы [23].

В настоящей работе полагается, что постоянная Планка $\hbar=1$.

\section{Теория и методы расчета}

Релятивистская спектр водородоподобного иона определяется стационарным уравнением Дирака:

$$
H \psi(\mathbf{r})=E \psi(\mathbf{r})
$$

и в присутствии однородного электрического поля гамильтониан имеет следующий вид:

$$
H=c(\boldsymbol{\alpha} \cdot \mathbf{p})+V_{\mathrm{nucl}}(r)-e F z+\beta m_{e} c^{2} .
$$

Здесь $e-$ заряд электрона $(e<0), c-$ скорость света, $(\boldsymbol{\alpha}, \beta)$ - матрицы Дирака, $V_{\text {nucl }}(r)$ - потенциал ядра, предполагается, что электрическое поле напряженности $F$ направлено вдоль оси $z$. В представленной работе для потенциала ядра используется модель равномерно заряженного шара:

$$
V_{\text {nucl }}(r)= \begin{cases}\frac{e Z}{2 R_{\text {nucl }}}\left(3-\frac{r^{2}}{R_{\text {nucl }}^{2}}\right), & r<R_{\text {nucl }}, \\ \frac{e Z}{r}, & r>R_{\text {nucl }}\end{cases}
$$

где $R_{\text {nucl }}$ - радиус ядра.

Уравнение Дирака рассматривается в сферической системе координат $(r, \theta, \varphi)$. Гамильтониан (2) обладает аксиальной симметрией относительно оси $z$, что приводит к сохранению проекции $m$ полного углового момента на эту ось и дает возможность отделить азимутальный угол $\varphi$ от остальных координат. Разделение переменных можно осуществить путем подстановки функции вида

$$
\psi_{m}(r, \theta, \varphi)=\frac{1}{r}\left(\begin{array}{c}
G_{1}(r, \theta) \exp \left[i\left(m-\frac{1}{2}\right) \varphi\right] \\
G_{2}(r, \theta) \exp \left[i\left(m+\frac{1}{2}\right) \varphi\right] \\
i F_{1}(r, \theta) \exp \left[i\left(m-\frac{1}{2}\right) \varphi\right] \\
i F_{2}(r, \theta) \exp \left[i\left(m+\frac{1}{2}\right) \varphi\right]
\end{array}\right)
$$

в уравнение Дирака (1). После такой подстановки уравнение Дирака можно привести к следующей форме:

$$
H_{m} \Phi(r, \theta)=E \Phi(r, \theta) .
$$

Здесь четырехкомпонентная волновая функция $\Phi(r, \theta)$ имеет вид

$$
\Phi(r, \theta)=\left(\begin{array}{c}
G_{1}(r, \theta) \\
G_{2}(r, \theta) \\
F_{1}(r, \theta) \\
F_{2}(r, \theta)
\end{array}\right)
$$

а гамильтониан $H_{m}$ можно выразить как

$$
\begin{aligned}
& H_{m}=\left(\begin{array}{cc}
m_{e} c^{2}+V_{\mathrm{nucl}}+e F z & c D_{m} \\
-c D_{m} & -m_{e} c^{2}+V_{\mathrm{nucl}}+e F z
\end{array}\right), \\
& D_{m}=\left(\sigma_{z} \cos \theta+\sigma_{x} \sin \theta\right)\left(\frac{\partial}{\partial r}-\frac{1}{r}\right) \\
& +\frac{1}{r}\left(\sigma_{x} \cos \theta-\sigma_{z} \sin \theta\right) \frac{\partial}{\partial \theta} \\
& +\frac{1}{r \sin \theta}\left(i m \sigma_{y}+\frac{1}{2} \sigma_{x}\right),
\end{aligned}
$$

где $\sigma_{x}, \sigma_{y}$ и $\sigma_{z}$ представляют собой матрицы Паули.

Из-за присутствия однородного электрического поля $F$ в спектре гамильтониана (7) отсутствуют связанные состояния. Зато там присутствуют резонансы, у которых есть ненулевая ширина $\Gamma$, определяющая вероятность ионизации электрона. Так как резонансные состояния не принадлежат гильбертову пространству, они не могут быть описаны квадратично интегрируемыми базисными функциями. Для того, чтобы преодолеть данную проблему, используется метод комплексного вращения, основанный на повороте радиальной координаты в комплексную плоскость. Вследствие такого поворота волновые функции резонансов становятся квадратично интегрируемыми, что дает возможность использовать стандартные методы конечного базисного набора. В представленной работе используется так называемое внешнее комплексное вращение (exterior complex scaling, ECS), согласно которому радиальная координата преобразуется следующим образом:

$$
r \rightarrow \begin{cases}r, & r \leq r_{0}, \\ r_{0}+\left(r-r_{0}\right) e^{i \Theta}, & r>r_{0},\end{cases}
$$

где параметр $r_{0}$ выбирается так, чтобы при $r>r_{0}$ рассматриваемый потенциал представлял собой аналитическую функцию. Таким образом, для потенциала (3) должно выполняться условие $r_{0} \geq R_{\text {nucl }}$. Во всех расчетах, результаты которых представлены в данной статье, полагалось $r_{0}=R_{\text {nucl. }}$.

После преобразования (9) собственные энергии гамильтониана (7), соответствующие резонансам, принимают комплексные значения:

$$
E_{\mathrm{CS}}=E-i \Gamma / 2 \text {. }
$$


Таблица 1. Положение $E$ и ширина $Г$ основного состояния атома водорода в однородном электрическом поле как функции напряженности поля $F$

\begin{tabular}{|c|c|c|c|c|c|}
\hline \multirow{2}{*}{$F$, a.u. } & \multirow{2}{*}{ Ref. } & \multicolumn{2}{|c|}{ Релятивистские } & \multicolumn{2}{|c|}{ Нерелятивистские } \\
\hline & & $E$, a.u. & $\Gamma$, a.u. & $E$, a.u. & $\Gamma$, a.u. \\
\hline 0.01 & $\mathrm{a}$ & & & & \\
\hline \multirow[t]{2}{*}{0.02} & $\begin{array}{l}\mathrm{b} \\
\mathrm{a}\end{array}$ & $\begin{array}{l}-0.50023223 \\
-0.50091583(1)\end{array}$ & & -0.5 & 0 \\
\hline & $\mathrm{b}$ & -0.50091585 & & -0.5 & 0 \\
\hline \multirow[t]{2}{*}{0.03} & $\begin{array}{l}\mathrm{a} \\
\mathrm{b}\end{array}$ & $\begin{array}{l}-0.50208081(1) \\
-0.50208083\end{array}$ & $2.236(2) \cdot 10^{-8}$ & -0.50207427 & \\
\hline & $\mathrm{c}$ & & & -0.50207427 & $2.23752 \cdot 10^{-8}$ \\
\hline \multirow[t]{2}{*}{0.04} & a & $-0.50377803(1)$ & $3.8913(9) \cdot 10^{-6}$ & & \\
\hline & $\begin{array}{l}\mathrm{b} \\
\mathrm{a}\end{array}$ & $\begin{array}{l}-0.50377804 \\
-0.50611171(1)\end{array}$ & $\begin{array}{l}4.1 \cdot 10 \\
7.7163(1) \cdot 10^{-5}\end{array}$ & -0.50377159 & $3.9 \cdot 10^{-6}$ \\
\hline 0.05 & $\mathrm{~b}$ & $\begin{array}{l}-0.50611173 \\
-0.50920954(1)\end{array}$ & $\begin{array}{l}7.74 \cdot 10^{-5} \\
51496(1) \cdot 10^{-4}\end{array}$ & -0.50610543 & $7.72 \cdot 10^{-6}$ \\
\hline 0.06 & $\begin{array}{l}\mathrm{a} \\
\mathrm{b}\end{array}$ & $\begin{array}{l}-0.50920954(1) \\
-0.50920955\end{array}$ & $\begin{array}{l}5.1496(1) \cdot 10^{-4} \\
5.152 \cdot 10^{-4}\end{array}$ & -0.50920345 & $5.151 \cdot 10^{-4}$ \\
\hline \multirow[t]{2}{*}{0.07} & $\mathrm{a}$ & $-0.51308262(1)$ & $1.8470(1) \cdot 10^{-3}$ & & $5.151 \cdot 10$ \\
\hline & $\begin{array}{l}\mathrm{b} \\
\mathrm{a}\end{array}$ & $\begin{array}{l}-0.51308260 \\
-0.51756625(1)\end{array}$ & $\begin{array}{l}1.8473 \cdot 10^{-3} \\
4.53896(3) \cdot 10^{-3}\end{array}$ & -0.51307677 & $1.8474 \cdot 10^{-3}$ \\
\hline 0.08 & $\mathrm{~b}$ & -0.51756619 & $4.5391 \cdot 10^{-3}$ & -0.51756062 & $4.5397 \cdot 10^{-2}$ \\
\hline \multirow[t]{2}{*}{0.09} & $\mathrm{a}$ & $-0.52241824(1)$ & $8.78282(2) \cdot 10^{-3}$ & & \\
\hline & $\mathrm{b}$ & -0.52241821 & $8.7828 \cdot 10^{-3}$ & -0.52241281 & $8.7840 \cdot 10^{-3}$ \\
\hline \multirow[t]{2}{*}{0.1} & a & $-0.52742345(1)$ & $1.453646(3) \cdot 10^{-2}$ & & \\
\hline & $\mathrm{b}$ & -0.527423540 & $1.45362 \cdot 10^{-2}$ & -0.52741818 & $1.45381 \cdot 10^{-2}$ \\
\hline
\end{tabular}

Примечание. а - данная работа, $\mathrm{b}$ - работа [23], $\mathrm{c}$ - работа [18].

Действительная часть $E$ этой величины соответствует положению резонанса, а мнимая - определяет его ширину $\Gamma$.

Решения повернутого в комплексную плоскость уравнения Дирака находятся с помощью конечного базисного набора. Волновая функция $\Phi(r, \theta)$ раскладывается по базису:

$$
\Phi(r, \theta)=\sum_{n=1}^{N} C_{n} W_{n}(r, \theta) .
$$

Базисные функции $W_{n}(r, \theta)$ конструируются из Всплайнов согласно методу дуального кинетического баланса (ДКБ) для систем с аксиальной симметрией [28]. Техника ДКБ предотвращает появление в спектре шпуриозных состояний $[40,41]$. Подробное описание способа построения базиса можно найти в работе [42]. Подстановкой разложения (11) уравнение Дирака (5) сводится к конечномерной обобщенной задаче на собственные значения, которая решается с помощью стандартных численных процедур.

\section{Результаты и обсуждение}

В представленной работе рассматривались только состояния с проекцией полного углового момента $m=1 / 2$. Комплексные значения энергии $E_{\mathrm{CS}}$ были получены с помощью метода, описанного выше. Положения и ширины резонансов находятся из значения $E_{\mathrm{CS}}$ согласно формуле (10).

Точные решения повернутого уравнения Дирака не зависят от угла комплексного вращения $\Theta$. Однако решения, полученные в конечном базисе, такую зависимость имеют. Для улучшения сходимости по количеству базисных функций целесообразно проводить расчеты при оптимальном угле комплексного вращения $\Theta_{\text {opt }}$, который соответствует минимуму производной $|d E / d \Theta|$. Необходимо отметить, что для разных состояний $\Theta_{\mathrm{opt}}$ принимает разные значения.

Расчеты производились в два этапа. На первом этапе с помощью расчетов в базисе небольшого размера находились оптимальные углы $\Theta_{\text {opt. }}$ На втором этапе вычисления осуществлялись уже только при оптимальных значениях угла комплексного вращения. При этом расчеты проводились с базисами различных размеров с целью определить погрешность итоговых значений. Подробно используемая методика определения погрешностей описана в работах $[32,33]$.

Результаты, полученные для основного состояния атома водорода, представлены в табл. 1. Для сравнения также приведены соответствующие релятивистские и нерелятивистские значения из статей $[18,23]$. Как можно видеть из таблицы, результаты, полученные в данной работе, неплохо согласуются со значениями из статьи [23] при большой напряженности поля $F$. Однако при относительно слабом поле результаты для 
Таблица 2. Положения $E$ и ширины $Г$ нижних возбужденных состояний водородоподобного иона $(Z=10)$ в однородном электрическом поле как функции напряженности поля $F$

\begin{tabular}{|c|c|c|c|c|c|}
\hline \multirow{2}{*}{$F$, a.u. } & \multirow{2}{*}{ Ref. } & \multicolumn{2}{|c|}{ Релятивистские } & \multicolumn{2}{|c|}{ Нерелятивистские } \\
\hline & & $E$, a.u. & $\Gamma$, a.u. & $E$, a.u. & $\Gamma$, a.u. \\
\hline \multicolumn{6}{|c|}{$2 s_{1 / 2}$} \\
\hline $\begin{array}{l}2 \\
4 \\
6 \\
8 \\
10\end{array}$ & $\begin{array}{l}\mathrm{a} \\
\mathrm{b} \\
\mathrm{a} \\
\mathrm{b} \\
\mathrm{a} \\
\mathrm{b} \\
\mathrm{a} \\
\mathrm{b} \\
\mathrm{a} \\
\mathrm{b}\end{array}$ & $\begin{array}{l}-12.546703(1) \\
-12.54670 \\
-12.646250(1) \\
-12.64625 \\
-12.840441(1) \\
-12.84044 \\
-13.138005(1) \\
-13.13801 \\
-13.463513(1) \\
-13.46349\end{array}$ & $\begin{array}{l}7.790(1) \cdot 10^{-5} \\
8 \cdot 10^{-5} \\
2.1280(1) \cdot 10^{-2} \\
2.126 \cdot 10^{-2} \\
2.04167(2) \cdot 10^{-1} \\
2.0423 \cdot 10^{-1} \\
6.21217(1) \cdot 10^{-1} \\
6.214 \cdot 10^{-1}\end{array}$ & $\begin{array}{l}-12.53157 \\
-12.63169 \\
-12.82715 \\
-13.12615 \\
-13.45247\end{array}$ & $\begin{array}{l}0 \\
8 \cdot 10^{-5} \\
2.178 \cdot 10^{-2} \\
2.0731 \cdot 10^{-1} \\
6.2792 \cdot 10^{-1}\end{array}$ \\
\hline \multicolumn{6}{|c|}{$2 p_{1 / 2}$} \\
\hline $\begin{array}{l}2 \\
4 \\
6 \\
8 \\
10\end{array}$ & $\begin{array}{l}\mathrm{a} \\
\mathrm{b} \\
\mathrm{a} \\
\mathrm{b} \\
\mathrm{a} \\
\mathrm{b} \\
\mathrm{a} \\
\mathrm{b} \\
\mathrm{a} \\
\mathrm{b}\end{array}$ & $\begin{array}{l}-13.149636(1) \\
-13.14961 \\
-13.867642(1) \\
-13.86772 \\
-14.719703(1) \\
-14.71966 \\
-15.674145(1) \\
-15.67420 \\
-16.616918(1) \\
-16.61683 \\
\end{array}$ & $\begin{array}{l}2 \cdot 10^{-5} \\
4.2833(4) \cdot 10^{-4} \\
4.4 \cdot 10^{-4} \\
6.5523(1) \cdot 10^{-2} \\
6.554 \cdot 10^{-2} \\
4.27531(1) \cdot 10^{-1} \\
4.2773 \cdot 10^{-1} \\
1.08062(1) \\
1.08039\end{array}$ & $\begin{array}{l}-13.13533 \\
-13.85488 \\
-14.70918 \\
-15.66554 \\
-16.60929 \\
\end{array}$ & $\begin{array}{l}1 \cdot 10^{-5} \\
4.5 \cdot 10^{-4} \\
6.676 \cdot 10^{-2} \\
4.3223 \cdot 10^{-1} \\
1.08833\end{array}$ \\
\hline \multicolumn{6}{|c|}{$2 p_{3 / 2}$} \\
\hline $\begin{array}{l}2 \\
4 \\
6 \\
8\end{array}$ & $\begin{array}{l}\mathrm{a} \\
\mathrm{b} \\
\mathrm{a} \\
\mathrm{b} \\
\mathrm{a} \\
\mathrm{b} \\
\mathrm{a} \\
\mathrm{b} \\
\mathrm{a} \\
\mathrm{b}\end{array}$ & $\begin{array}{l}-11.948679(1) \\
-11.94869 \\
-11.446913(1) \\
-11.44692 \\
-11.019910(1) \\
-11.01991 \\
-10.683798(1) \\
-10.68390 \\
-10.404716(1) \\
-10.40443\end{array}$ & $\begin{array}{l}2 \cdot 10^{-5} \\
1.426(2) \cdot 10^{-5} \\
3 \cdot 10^{-5} \\
5.9228(6) \cdot 10^{-3} \\
5.93 \cdot 10^{-3} \\
8.4307(3) \cdot 10^{-2} \\
8.444 \cdot 10^{-2} \\
3.23139(1) \cdot 10^{-1} \\
3.2374\end{array}$ & $\begin{array}{l}-11.43054 \\
-11.00366 \\
-10.66829 \\
-10.38915\end{array}$ & $\begin{array}{l}1 \cdot 10^{-5} \\
2 \cdot 10^{-5} \\
6.09 \cdot 10^{-3} \\
8.619 \cdot 10^{-2} \\
3.2853\end{array}$ \\
\hline
\end{tabular}

Примечание. а - данная работа, $\mathrm{b}$ - работа $[23]$.

ширины систематически меньше релятивистских значений из работы [23] и ближе к нерелятивистским значениям. При $F<0.03$ a.u. точности используемого метода недостаточно, чтобы получить значения для ширины.

В табл. 2 приведены результаты для состояний $2 s$, $2 p_{1 / 2}$ и $2 p_{3 / 2}$ водородоподобного иона неона $(Z=10)$. Для некоторых значений $F$ есть небольшие расхождения в положении резонансов между данной работой и статьей [23]. Стоит отметить, что в работе [23] использовалась точечная модель ядра, в то время как в представленных расчетах применяется модель равномерно заряженного шара, что позволяет учесть конечный размер ядра. Однако влияние конечного размера ядра в данном случае незначительно и, хотя в какой-то степени ответственно за различие в положениях резонансов, не может объяснить существенное отличие в релятивистских значениях ширины. Из таблицы видно, что полученные в данной работе результаты для ширины всегда меньше нерелятивистских. Более того, относительная разность между релятивистскими и нерелятивистскими результатами возрастает с уменьшением поля. При этом значения из работы [23] при уменьшении поля в какойто момент начинают демонстрировать обратную динамику, и релятивистское значение ширины становится больше нерелятивистского. Необходимо отметить, что относительное уменьшение релятивистского значения ширины с ослаблением поля согласуется с выводами 
из работ [21,22], сделанными на основе расчетов в квазиклассическом приближении.

\section{Заключение}

Выполнены релятивистские расчеты положений и ширин нижних состояний атома водорода и водородоподобного иона неона в однородном электрическом поле. Вычисления были проведены с помощью метода конечного базисного набора с использованием техники комплексного вращения. Часть полученных результатов не согласуется с соответствующими значениями из работы [23]. Причина расхождения неясна. Все полученные релятивистские значения для ширины меньше соответствующих нерелятивистских, и относительная разность увеличивается с уменьшением напряженности электрического. Качественно данный результат согласуется с выводами работ [21,22], полученными в рамках квазиклассического приближения.

\section{Финансирование работы}

Исследования проведены при финансовой поддержке гранта Президента Российской Федерации № МK-1626.2020.2. Работа И.А.М. также была поддержана фондом развития теоретической физики и математики „БАЗИС“. Расчеты были проведены на оборудовании „Вычислительного центра СПбГУ“.

\section{Конфликт интересов}

Авторы заявляют, что у них нет конфликта интересов.

\section{Список литературы}

[1] H. Rausch v. Traubenberg, R. Gebauer, G. Lewin. Naturwissenschaften, 18, 417 (1930). DOI: $10.1007 / \mathrm{BF} 01501125$

[2] R.F. Stebbings. Science, 193, 537 (1976). DOI: $10.2307 / 1742745$

[3] M.G. Littman, M.L. Zimmerman, D. Kleppner. Phys. Rev. Lett., 37, 486 (1976). DOI: 10.1103/PhysRevLett.37.486

[4] P.M. Koch, D.R. Mariani. Phys. Rev. Lett., 46, 1275 (1981). DOI: 10.1103/PhysRevLett.46.1275

[5] T. Bergeman, C. Harvey, K.B. Butterfield, H.C. Bryant, D.A. Clark, P.A.M. Gram, D. MacArthur, M. Davis, J.B. Donahue, J. Dayton, W.W. Smith. Phys. Rev. Lett., 53, 775 (1984). DOI: 10.1103/PhysRevLett.53.775

[6] A.S. Stodolna, A. Rouzée, F.Lépine, S. Cohen, F. Robicheaux, A. Gijsbertsen, J.H. Jungmann, C. Bordas, M.J.J. Vrakking. Phys. Rev. Lett., 110, 213001 (2013). DOI: 10.1103/PhysRevLett.110.213001

[7] M. Hehenberger, H.V. Mc Intosh, E. Brändas. Phys. Rev. A, 10, 1494 (1974). DOI: 10.1103/PhysRevA.10.1494

[8] С.А. Запрягаев. Опт. и спектр., 44, 892 (1978). [S.A. Zapryagaev. Opt. Spectrosc., 44892 (1978).].

[9] L. Benassi, V. Grecchi. J. Phys. B, 13, 911 (1980). DOI: $10.1088 / 0022-3700 / 13 / 5 / 016$
[10] D. Farrelly, W. P. Reinhardt. J. Phys. B, 16, 2103 (1983). DOI: $10.1088 / 0022-3700 / 16 / 12 / 008$

[11] J.A.C. Gallas, H. Walther, E. Werner. Phys. Rev. A, 26, 1775 (1982). DOI: 10.1103/PhysRevA.26.1775

[12] R.J. Damburg, V.V. Kolosov. J. Phys. B, 9, 3149 (1976). DOI: $10.1088 / 0022-3700 / 9 / 18 / 006$

[13] R.J. Damburg, V.V. Kolosov. J. Phys. B, 11, 1921 (1978). DOI: $10.1088 / 0022-3700 / 11 / 11 / 009$

[14] V.V. Kolosov. J. Phys. B, 20, 2359 (1987). DOI: $10.1088 / 0022-3700 / 20 / 11 / 008$

[15] C.S. Lai. Phys. Lett. A, 83, 322 (1981). DOI: $10.1016 / 0375-9601(81) 90150-X$

[16] V.V. Kolosov. J. Phys. B, 16, 25 (1983). DOI: $10.1088 / 0022-3700 / 16 / 1 / 004$

[17] C.Y. Lin, Y. K. Ho. J. Phys. B, 44, 175001 (2011). DOI: $10.1088 / 0953-4075 / 44 / 17 / 175001$

[18] J. Rao, W. Liu, B. Li. Phys. Rev. A, 50, 1916 (1994). DOI: 10.1103/PhysRevA.50.1916

[19] F.M. Fernández. Phys. Rev. A, 54, 1206 (1996). DOI: 10.1103/PhysRevA.54.1206

[20] U.D. Jentschura. Phys. Rev. A, 64, 013403 (2001). DOI: 10.1103/PhysRevA.64.013403

[21] N. Milosevic, V.P. Krainov, T. Brabec. Phys. Rev. Lett., 89, 193001 (2002). DOI: 10.1103/PhysRevLett.89.193001

[22] N. Milosevic, V.P. Krainov, T. Brabec. J. Phys. B, 35, 3515 (2002). DOI: 10.1088/0953-4075/35/16/311

[23] I.A. Ivanov, Y.K. Ho. Phys. Rev. A, 69, 023407 (2004). DOI: 10.1103/PhysRevA.69.023407

[24] P.A. Batishchev, O.I. Tolstikhin, T. Morishita. Phys. Rev. A, 82, 023416 (2010). DOI: 10.1103/PhysRevA.82.023416

[25] L. Fernández-Menchero, H.P. Summers. Phys Rev. A, 88, 022509 (2013). DOI: 10.1103/PhysRevA.88.022509

[26] F.M. Fernández. Applied Mathematics and Computation, 317, 101 (2018). DOI: 10.1016/j.amc.2017.08.050

[27] A. Maquet, Shih-I Chu, W.P. Reinhardt. Phys. Rev. A, 27, 2946 (1983). DOI: 10.1103/PhysRevA.27.2946

[28] E.B. Rozenbaum, D.A. Glazov, V.M. Shabaev, K.E. Sosnova, D.A. Telnov. Phys. Rev. A, 89, 012514 (2014). DOI: 10.1103/PhysRevA.89.012514

[29] D. Budker, J.R. Crespo López-Urrutia, A. Derevianko, V.V. Flambaum, M.W. Krasny, A. Petrenko, S. Pustelny, A. Surzhykov, V.A. Yerokhin, M. Zolotorev. Annalen der Physik, 532, 2000204 (2020). DOI: 10.1002/andp.202000204

[30] S. Kieslich, S. Schippers, W. Shi, A. Müller, G. Gwinner, M. Schnell, A. Wolf, E. Lindroth, M. Tokman. Phys. Rev. A, 70, 0042714 (2004). DOI: 10.1103/PhysRevA.70.042714

[31] A. Müller, E. Lindroth, S. Bari, A. Borovik Jr., P.-M. Hillenbrand, K. Holste, P. Indelicato, A.L.D. Kilcoyne, S. Klumpp, M. Martins, J. Viefhaus, P. Wilhelm, S. Schippers. Phys. Rev. A, 98, 033416 (2018). DOI: $10.1103 /$ PhysRevA.98.033416

[32] V.A. Zaytsev, I.A. Maltsev, I.I. Tupitsyn, V.M. Shabaev. Phys. Rev. A, 100, 052504 (2019). DOI: 10.1103/PhysRevA.100.052504

[33] В.А. Зайцев, И.А. Мальцев, И.И. Тупицын, В.М. Шабаев, В.Ю. Иванов. Опт. и спектр. 128, 318 (2020). DOI: $10.21883 /$ OS.2020.03.49057.329-19 [V.A. Zaytsev, I.A. Maltsev, I.I Tupitsyn, V.M. Shabaev, V.Y. Ivanov. Opt. Spectrosc., 128, 307 (2020). DOI: $10.1134 / \mathrm{S} 0030400 \mathrm{X} 20030200]$.

[34] E. Ackad, M. Horbatsch. Phys. Rev. A, 75, 022508 (2007). DOI: $10.1103 /$ PhysRevA.75.022508 
[35] E. Ackad, M. Horbatsch. Phys. Rev. A, 76, 022503 (2007). DOI: $10.1103 /$ PhysRevA.76.022503

[36] A. Marsman, M. Horbatsch. Phys. Rev. A, 84, 032517 (2011). DOI: $10.1103 /$ PhysRevA.84.032517

[37] И.А. Мальцев, В.М. Шабаев, В.А. Зайцев, Р.В. Попов, Д.А. Тумаков. Опт. и спектр., 129, 1094 (2020). DOI: $\quad 10.21883 /$ OS.2020.08.49703.117-20 [I.A. Maltsev, V.M. Shabaev, V.A. Zaytsev, R.V. Popov, D.A Tumakov. Opt. Spectrosc., 128, 1100 (2020).

DOI: $10.1134 / \mathrm{S} 0030400 X 2008024 \mathrm{X}]$.

[38] N. Moiseyev. Phys. Rep., 302, 211 (1998). DOI: $10.1016 / \mathrm{S} 0370-1573(98) 00002-7$

[39] E. Lindroth, L. Argenti. Adv. Quantum Chem., 63, 247 (2012). DOI: 10.1016/B978-0-12-397009-1.00005-9

[40] V.M. Shabaev, I.I. Tupitsyn, V.A. Yerokhin, G. Plunien, G. Soff. Phys. Rev. Lett., 93, 130405 (2004). DOI: 10.1103/PhysRevLett.93.130405

[41] И.И. Тупицын, В.М. Шабаев. Опт. и спектр., 105, 203 (2008). [I.I. Tupitsyn, V.M. Shabaev. Opt. Spectrosc., 105, 183 (2008). DOI: 10.1134/S0030400X08080043]

[42] I.A. Maltsev, V.M. Shabaev, R.V. Popov, Y.S. Kozhedub, G. Plunien, X. Ma, Th. Stöhlker. Phys. Rev. A, 98, 062709 (2018). DOI: 10.1103/PhysRevA.98.062709 